\title{
Multi loop Controller Design for Multivariable Processes Employing SISO PI Tuning Rules
}

\author{
G. Vamsi Krishna, Naregalkar Akshay
}

\begin{abstract}
Ability of the proportional integral (PI) and proportional integral derivative (PID) controllers in set point tracking and disturbance rejection has led to their wide usage in industrial applications. The desired controller performance can be achieved by application of suitable tuning rules. The presence of interacting loops in a process makes it a challenging task of PI controllers design in multivariable processes. This problem highly mitigated by employing simple single input single output internal model controller (IMC). This IMC PI controller values are applied for different distillation process involving distillation columns like Wardle and Wood $(\mathrm{WW})$, Wood and Berry (WB) and Vinate-Luyben $(V-L)$. The obtained simulation with proposed method gives better results and performance indices values compared to other control tuning strategies.
\end{abstract}

Keywords- PI Controller; tuning, ;mulivariable process ;IMC ; $S I M C ; B L T$

\section{INTRODUCTION}

Most of the process control industries are multi input multi output (MIMO) in nature. Performance characteristic of a controller are set point tracking, disturbance rejection and robustness. Achieving these performance characteristics for multi variable processes with satisfactory controller performance is not easy. So it is always challengeable task for control system engineers. MIMO processes are mainly suffering with interaction effect between input and output (i-o) variables.

This interaction effect overcome or minimized by proper selection of pairing between the control loops. Best pairing selected by using knowledge of relative gain array (RGA). Control loops are great extent controllable by adopting PI/PID controllers. In present century, 90 to $95 \%$ multi variable processes control loops are controlling by PI control strategies.

Since Past decade many articles are submitted to design multi variable processes adopting PI Controllers. Broadly it is able to divide in to multi loop control strategies, decouplers and centralized controllers.

Decoupler based controllers, available in ideal [1], normalized [2], Simplified [3] and inverted [4]. The advantages of decoupler control strategies are best suitable for moderate and severe interaction effect, It allows usage of SISO controller tuning methodologies, more fault tolerance
- Any fault occur in one control loop that loop only effected other loops are in safe. Disadvantage of these methods are

- Design decoupler for high dimensional process is includes huge mathematical equations

- If Process transfer function matrix (TFM) contains higher values of time delays compare to interaction time delay, design of decoupler is not easy.

These two problems are overcome by using centralized and decentralised control strategies.

In full matrix control strategy, controller matrix is designed for both diagonal and off diagonal elements and then applied in centralised control strategies. In this approach PI/PID controllers are designed for each loop independently. Where as in some tuning techniques, calculation of PI/PID controller parameter separately for diagonal and off diagonal interaction terms. This method is suitable for both moderate or significant interaction effects situtaion. It is applicable for higher order MIMO systems also. The main problem of this centralised control strategy design is estimating filter factor is typical to calculate.

This filter factor is easily calculated by using multi loop control strategy. In this, MIMO process is treated as collection of multi single loops in decentralised control strategy. In detuning factor based methods, controller is designed for each individual loop separately taking interaction effect into account[5], [6], [7]. But it fails in getting desired servo and regulatory response in case of interaction effect is moderate and severe. Multi loop control strategies are best suitable for less interaction MIMO process design.

\section{MULTI LOOP CONTROL OF MIMO PROCESSES}

Multi loop controller techniques with decentralized controller mode are widely used in industries due to their simple structure, fewer tuning parameters and robustness. The specified block diagram for $2 \times 2$ MIMO process with multi loop controllers is shown in figure-1.

Revised Manuscript Received on April 12, 2019.

G. Vamsi Krishna,Department of EIE, VNR VignanaJyothi Institute of Engineering \& Technology, Hyderabad, Telangana, India (E-mail: vamsikrishna_g@vnrvjiet.in)

NaregalkarAkshay,Department of EIE, VNR VignanaJyothi Institute of Engineering \& Technology, Hyderabad, Telangana, India (E-mail: akshay_n@vnrvjiet.in) 


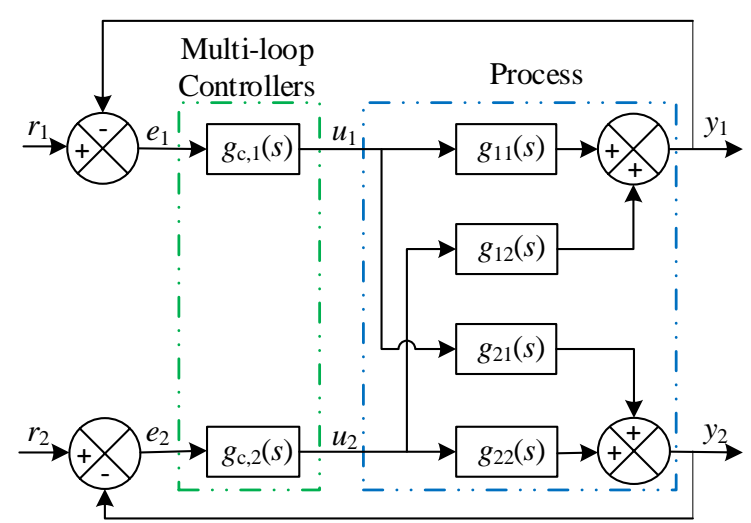

Fig.1: Block diagram of multi loop controller for $2 \times 2$ MIMO process

For a multi loop decentralized MIMO system with fixed control structure, the individual controller performance is determined mainly by tuning it separately. But, the presence of interaction between the control loops makes the tuning of multi loop PID controllers quite difficult. Very few tuning methods like Biggest logarithm tuning (BLT),Nyquist stability, IMC are available for the multi loop PID controllers but it burdens in high complex calculation and more iterative steps[8], [9], ,10]. The analytical tuning rule method, IMC is practical in order to solve above problem, but involves mathematical complexity in the multi loop systems problems.

\section{MULTIVARIABLE PROCESSES UNDER STUDY - DISTILLATION COLUMN}

In every industrial process sensed (measured output) variable taken as process variables, PID controller input/output variables are called as manipulated and control variables and desired response output called as set point. Normally controller design for Single Input and Single Output (SISO) systems is easy due to absence of interaction effect. Where as in Multi Input Multi Output (MIMO) processes, the controller design become difficult due to process interactions involving interaction of each manipulated variable with all other control variables, several measurements and control signal. Thus this interaction between several SISO loops makes the task of designing multi-loop controllers difficult.

\section{A. Wardle and Wood $(W W)$ distillation Column}

Wardle and Wood distillation Column (WW-DC) is used to separate water and ethanol and is described by equation 1 in the transfer function matrix $\mathrm{G}(\mathrm{s})$ form [8]. $G(s)=\left[\begin{array}{cc}\frac{0.126 e^{-6 s}}{60 s+1} & \frac{-0.101 e^{-12 s}}{(48 s+1)(45 s+1)} \\ \frac{0.094 e^{-8 s}}{38 s+1} & \frac{-0.12 e^{-8 s}}{35 s+1}\end{array}\right]$

\section{B. Wood and Berry (WB) distillation column}

Wood and Berry distillation column is a $2 \times 2$ binary distillation process separates methanol and water and is described by equation 2in transfer function matrix [11].

$$
G(s)=\left[\begin{array}{cc}
\frac{12.8 e^{-s}}{16.78 s+1} & \frac{-18.9 e^{-3 s}}{21 s+1} \\
\frac{6.6 e^{-7 s}}{10.9 s+1} & \frac{-19.4 e^{-3 s}}{14.4 s+1}
\end{array}\right]
$$

\section{Vinate-Luyben $(V-L)$ distillation column}

Vinate-Luyben (V-L) distillation is a $2 \times 2$ binary distillation process that separates the mixture of methanol and water and is described by equation 3 in transfer function matrix [8].

$$
G(s)=\left[\begin{array}{cc}
\frac{-2.2 e^{-s}}{2 s+1} & \frac{1.3 e^{-0.3 s}}{7 s+1} \\
\frac{-2.8 e^{-1.8 s}}{9.5 s+1} & \frac{4.3 e^{-0.35 s}}{9.2 s+1}
\end{array}\right]
$$

\section{PROPOSED METHOD}

The proposed work aims is to provide a simple, systematic and logical PID Controller design. This can be done by postulating a model for system under study, by forming desired control objectives and to obtain required controller parameters and structure. The desired controller parameters can be obtainedwith the Internal Model Control (IMC) structure [14].The model complexity and defined performance specification decide the IMC based controller design. This work demonstrates how to select controller design with proper tuning method to realize IMC based controller performance in chemical processes involving with first-order process plus lag.

Assume a first-order process plus lag process with transfer function expressed in equation 4

$$
G(s)=\frac{K_{m} e^{-\theta s}}{1+s T_{m}}
$$

The PI controller settings for this process are

$$
\begin{aligned}
& K_{P}=\frac{T_{m}}{\lambda K_{m}} \\
& T_{I}=T_{m}
\end{aligned}
$$

Where $\lambda$ is 1.7 times of lag or dead time.

The PI Controller parameters for different distillation column systems using the proposed and existing methods are shown in Table 1 .

TABLE I: Tuned Controller parameters for various MIMO distillation processes

\begin{tabular}{|l|l|l|l|l|}
\hline Process & Method & Controller & $k_{c}$ & $\tau_{I}$ \\
\hline \multirow{5}{*}{ WW } & \multirow{3}{*}{ BLT } & loop1 & 27.4 & 41.4 \\
\cline { 2 - 5 } & \multirow{3}{*}{ Z-N } & loop2 & -13.3 & 52.9 \\
\cline { 2 - 5 } & & loop1 & 71.42 & 19.98 \\
\cline { 2 - 5 } & \multirow{2}{*}{ IMC } & loop2 & -32.81 & 26.64 \\
\cline { 3 - 5 } & & loop1 & 49.0 & 63.0 \\
\hline & & loop2 & -23.88 & 39.0 \\
\hline
\end{tabular}




\begin{tabular}{|c|c|c|c|c|}
\hline & SIMC & $\begin{array}{l}\text { loop1 } \\
\text { 1oop2 }\end{array}$ & $\begin{array}{l}39.68 \\
-18.22\end{array}$ & $\begin{array}{l}48.0 \\
35.0\end{array}$ \\
\hline & Proposed & $\begin{array}{l}\text { loop1 } \\
\text { loop2 }\end{array}$ & $\begin{array}{l}46.68 \\
-21.44\end{array}$ & $\begin{array}{l}60.0 \\
35.0\end{array}$ \\
\hline \multirow{9}{*}{ WB } & \multirow{2}{*}{ BLT } & loop1 & 0.375 & 8.29 \\
\hline & & loop2 & 0.075 & 23.6 \\
\hline & \multirow{2}{*}{$\mathrm{Z}-\mathrm{N}$} & loop1 & 0.96 & 3.25 \\
\hline & & loop2 & -0.19 & 9.2 \\
\hline & \multirow{2}{*}{ IMC } & loop1 & 0.793 & 17.28 \\
\hline & & loop2 & -0.16 & 15.9 \\
\hline & \multirow{2}{*}{ SIMC } & loop1 & 0.655 & 8.0 \\
\hline & & loop2 & 0.123 & 14.4 \\
\hline & Proposed & $\begin{array}{l}\text { loop1 } \\
\text { loop2 }\end{array}$ & $\begin{array}{l}0.771 \\
-0.145\end{array}$ & $\begin{array}{l}16.78 \\
14.4\end{array}$ \\
\hline \multirow{8}{*}{ VL } & \multirow{2}{*}{ BLT } & loop1 & -1.07 & 7.1 \\
\hline & & loop2 & 1.97 & 2.58 \\
\hline & \multirow{2}{*}{$\mathrm{Z}-\mathrm{N}$} & loop1 & -2.4 & 3.16 \\
\hline & & loop2 & 4.45 & 1.15 \\
\hline & \multirow{2}{*}{ IMC } & loop1 & -2.0 & 7.5 \\
\hline & & loop2 & 3.66 & 9.37 \\
\hline & SIMC & $\begin{array}{l}\text { loop1 } \\
\text { loop2 }\end{array}$ & $\begin{array}{l}-1.59 \\
3.05\end{array}$ & $\begin{array}{l}7.0 \\
2.8\end{array}$ \\
\hline & Proposed & $\begin{array}{l}\text { loop1 } \\
\text { loop2 }\end{array}$ & $\begin{array}{l}-1.871 \\
3.595\end{array}$ & $\begin{array}{l}7.0 \\
9.2\end{array}$ \\
\hline
\end{tabular}

\section{SIMULATION RESULTS}

It can be observed from Table 2, that the proposed method gives better controller performance to meet desired specifications than other decentralized control design strategies .The comparison is done in terms of evaluation of various performance indices viz. integral absolute error (IAE), integral square error (ISE) . The $\mathrm{IAE}_{\mathrm{i}}$ and $\mathrm{ISE}_{\mathrm{i}}$ listed in Table II. Gives thesignificance of the IAE and ISE values for the applied step change at the $i^{\text {th }}$ set-point (SP), while the other SPs are maintained at zero. The ISE and IAE parameters are expressed in equation 6 and 7 , respectively.

$$
\begin{gathered}
I A E=\sum_{i=1}^{m} \int_{0}^{t}\left|e_{i}(t)\right| d t \\
I S E=\sum_{i=1}^{m} \int_{0}^{t} e_{i}^{2}(t) d t
\end{gathered}
$$

TABLE -II.Comparative analysis of Performance

\begin{tabular}{|c|c|c|c|c|}
\hline \multicolumn{5}{|l|}{ WB } \\
\hline & IAEi & ISEi & IAE & ISE \\
\hline \multirow{2}{*}{ BLT } & 35.32 & 12.78 & \multirow{2}{*}{56.35} & \multirow{2}{*}{19.39} \\
\hline & 21.03 & 6.60 & & \\
\hline \multirow{2}{*}{$\mathrm{Z}-\mathrm{N}$} & 92.98 & 35.86 & \multirow{2}{*}{202.86} & \multirow{2}{*}{86.04} \\
\hline & 109.88 & 50.17 & & \\
\hline \multirow{2}{*}{ IMC } & 14.96 & 6.30 & \multirow{2}{*}{27.60} & \multirow{2}{*}{11.13} \\
\hline & 12.64 & 4.83 & & \\
\hline \multirow{2}{*}{ SIMC } & 17.41 & 6.65 & \multirow{2}{*}{28.73} & \multirow{2}{*}{11.18} \\
\hline & 11.32 & 4.53 & & \\
\hline \multirow{2}{*}{ Proposed } & 14.00 & 6.09 & \multirow{2}{*}{25.22} & \multirow{2}{*}{10.72} \\
\hline & 11.21 & 4.62 & & \\
\hline \multicolumn{5}{|l|}{ VL } \\
\hline & IAEi & ISEi & IAE & ISE \\
\hline \multirow{2}{*}{ BLT } & 2.91 & 1.11 & \multirow{2}{*}{8.58} & \multirow{2}{*}{3.86} \\
\hline & 5.67 & 2.76 & & \\
\hline \multirow{2}{*}{$\mathrm{Z}-\mathrm{N}$} & 3.70 & 1.43 & \multirow{2}{*}{7.51} & \multirow{2}{*}{3.24} \\
\hline & 3.80 & 1.81 & & \\
\hline \multirow{2}{*}{ IMC } & 2.00 & 0.82 & \multirow{2}{*}{5.96} & \multirow{2}{*}{2.69} \\
\hline & 3.95 & 1.87 & & \\
\hline \multirow{2}{*}{ SIMC } & 2.37 & 0.93 & \multirow{2}{*}{6.33} & \multirow{2}{*}{2.98} \\
\hline & 3.96 & 2.05 & & \\
\hline Pronosed & 2.05 & 0.82 & 601 & 272 \\
\hline & 3.96 & 1.90 & & \\
\hline WW & & & & \\
\hline & IAEi & ISEi & IAE & ISE \\
\hline RI T & 89.88 & 27.64 & 17051 & 5756 \\
\hline DLI & 80.63 & 29.92 & 170.51 & ט.J.5 \\
\hline $7 \mathrm{~N}$ & 104.28 & 40.16 & 41010 & 35717 \\
\hline & 314.91 & 317.01 & & \\
\hline IMC & 53.36 & 15.58 & 11201 & 4152 \\
\hline & 59.56 & 25.94 & 112.71 & \\
\hline SIMC & 56.10 & 17.00 & 11288 & 4173 \\
\hline SIVIC & 56.78 & 24.72 & 112.00 & 41.15 \\
\hline Pronosed & 52.92 & 15.77 & 11108 & 4115 \\
\hline 19posed & 58.16 & 25.39 & 111.00 & 41.15 \\
\hline
\end{tabular}
indices values for various MIMO Distillation processes

MATLAB unit step response for $\mathrm{WW}$ and $\mathrm{WB}$ and VL are organized below fig.2,3 and 4 . 

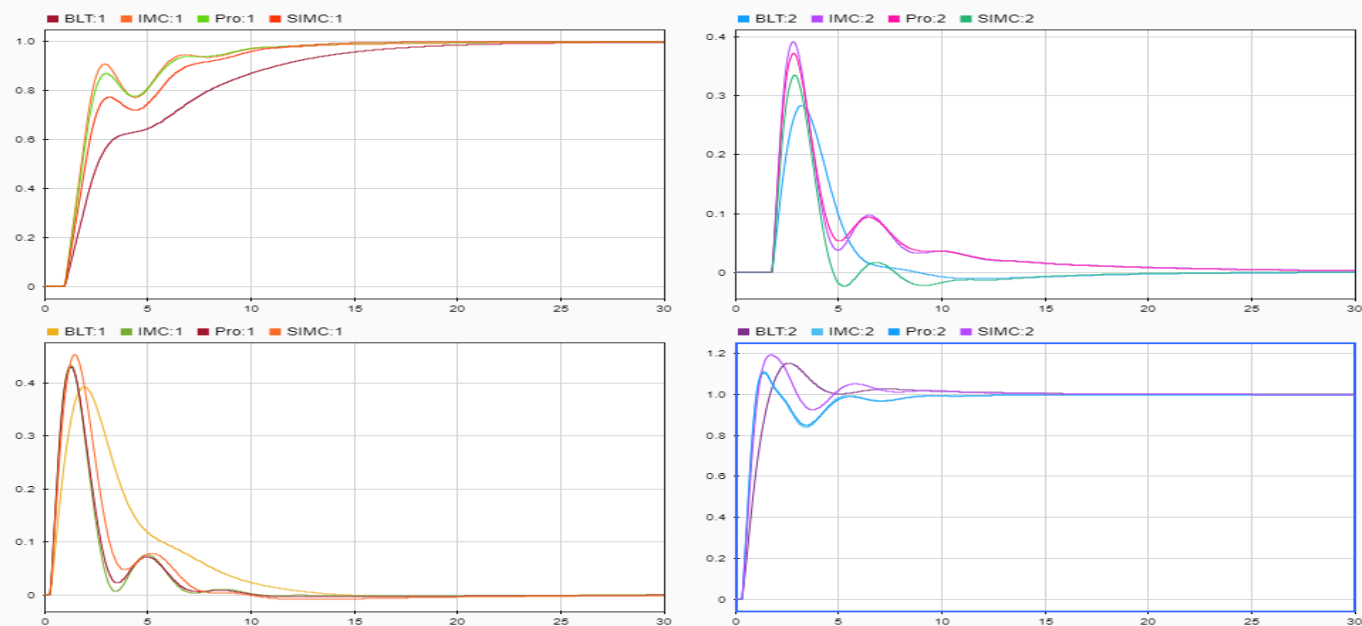

Fig.2. sequential step response for WW process
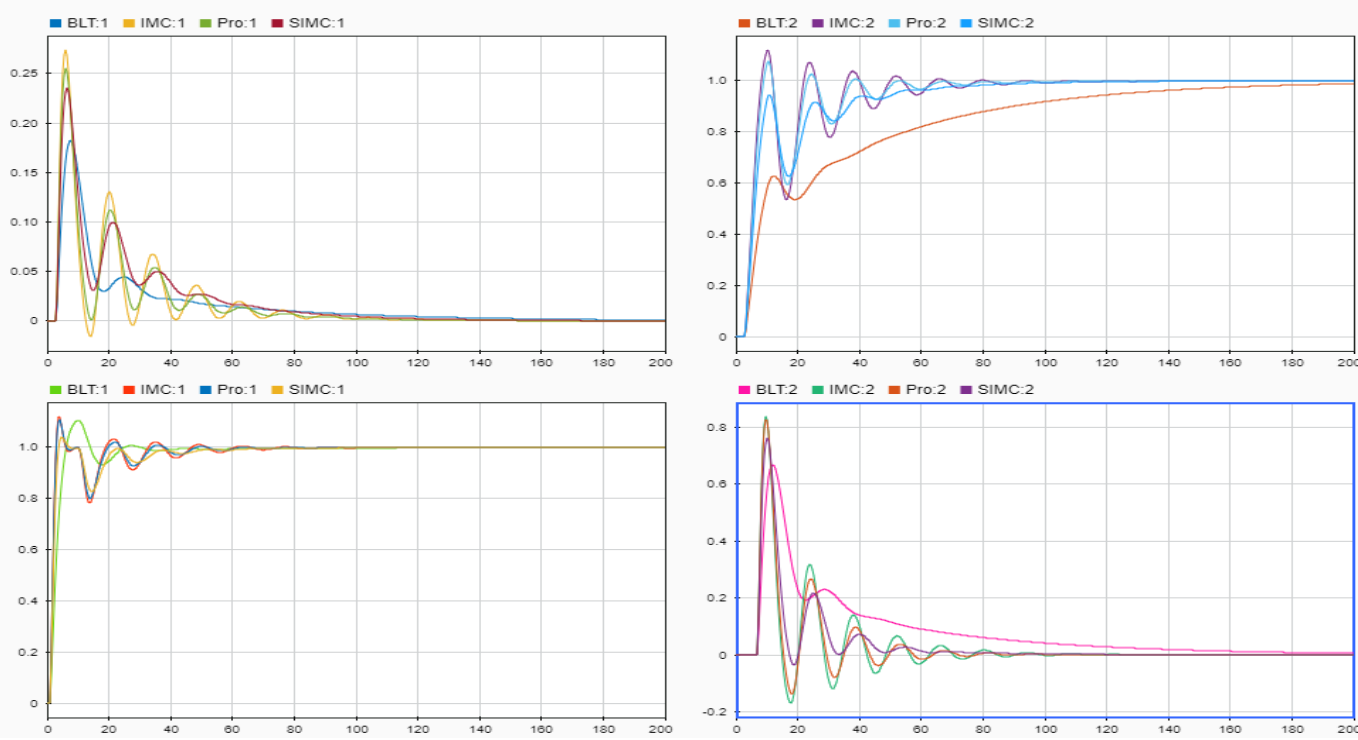

Fig.3. sequential step response for WB process
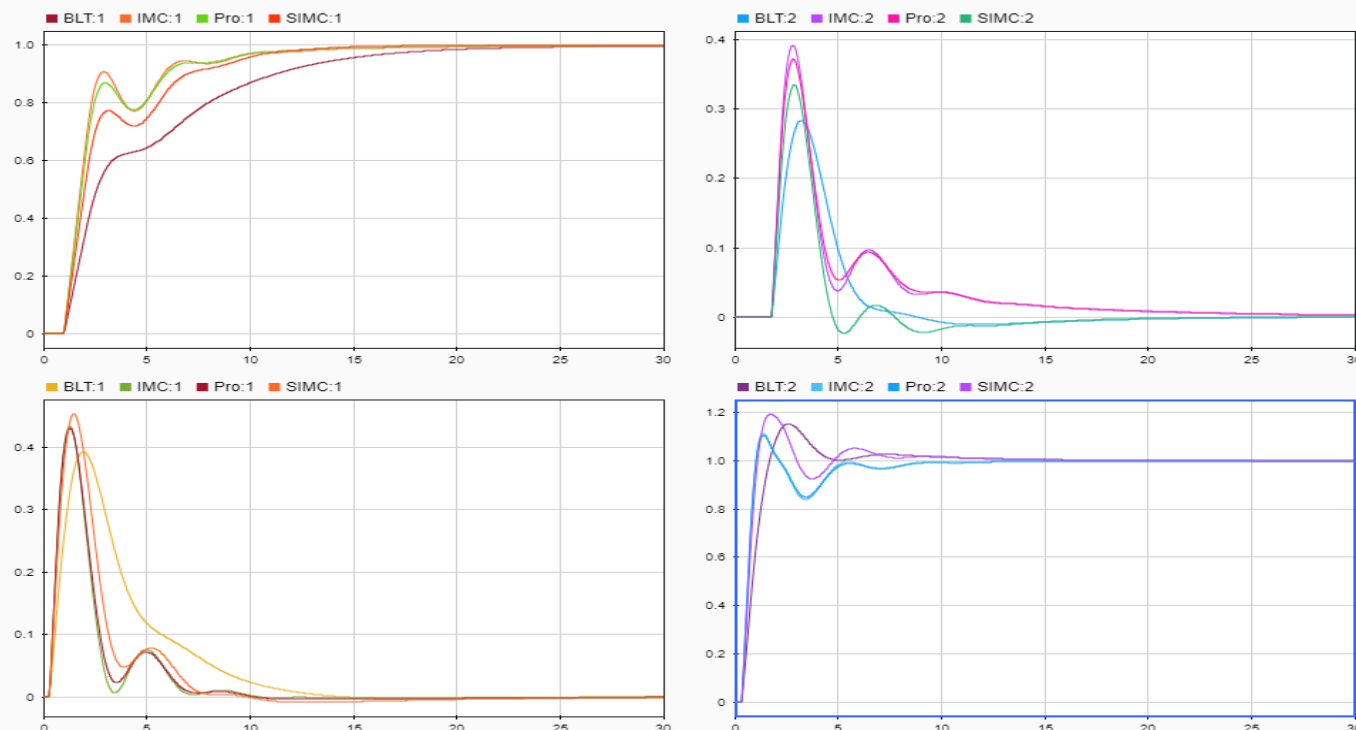

Fig.4. sequential step response for VL process

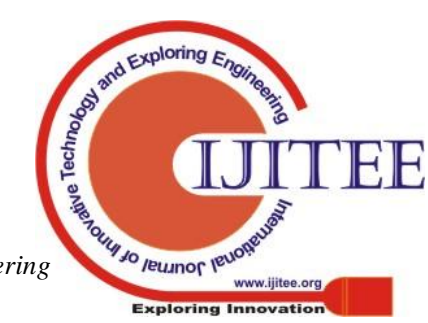




\section{CONCLUSION}

The proposed method with IMC tuning is used to design controllers for various MIMO Distillation Columns like Wardle and Wood (WW), Wood and Berry (WB)and Vinate-Luyben (V-L). It can be concluded from the simulation results and performance indices values that the proposed IMC tuning control algorithm gives better and satisfactory performance than the Ziegler - Nicholos and Biggest logarithm tuning and other IMC tuning control strategies. Thus the best results of desired controller specifications in terms of IAE and ISE are achieved by using the proposed method.

\section{REFERENCES}

1. W. L. Luyben, "Distillation decoupling," AIChE Journal, vol. 16, pp. 198-203, 1970.

2. Y. Shen, W.-J. Cai, and S. Li, "Normalized decoupling control for high-dimensional MIMO processes for application in room temperature control HVAC systems," Control Engineering Practice, vol. 18, pp. 652-664, 2010.

3. C. Rajapandiyan and M. Chidambaram, "Controller design for MIMO processes based on simple decoupled equivalent transfer functions and simplified decoupler," Industrial and Engineering ChemistryResearch, vol. 51, pp. 12398-12410, 2012.

4. J. Garrido, F. Vázquez, F. Morilla, and T. Hägglund, "Practical advantages of inverted decoupling," Proceedings of the Institution ofMechanical Engineers, Part I: Journal of Systems and Control, vol. 225, pp. 977-992, 2011.

5. T. N. L. Vu and M. Lee, "Independent design of multi-loop PI/PID controllers for interacting multivariable processes," Journal of ProcessControl, vol. 20, pp. 922-933, 2010.

6. Q. Xiong, W.-J. Cai, Effective transfer function method for decentralized control system design of multi-input multioutput processes, Journal of Process Control vol. 16, PP. 773784. 2006.

7. Q.-G. Wang, B. Zou, T.H. Lee, Q. Bi, Auto-tuning of multivariable PID controllers from decentralized relay feedback, Automatica 33, pp. 319-330. 1997.

8. W. L. Luyben, "Simple method for tuning SISO controllers in multivariable systems," Ind. Eng. Chem. Process Des. Dev., vol. 25, pp. 654-660, 1986.

9. D. Chen, D.E. Seborg, Design of decentralized PI control systems based on Nyquist stability analysis, Journal of Process Control 13, pp. 27-39, 2003.

10. S. Skogestad, "Simple analytic rules for model reduction and PID controller tuning," Journal of Process Control, vol. 13, pp. 291-309, 2003.

11. R. K. Wood and M. W. Berry, "Terminal composition control of a binary distillation column," Chemical Engineering Science, vol. 28, pp. 1707-1717, 1973.

12. G.H. Cohen and G.A. Coon, "Theoretical Consideration of Retarded Control," Trans. ASME, 75, pp. 827-834, 1953.

13. Smith, C. L. "Digital Computer Process Control"; Intext Educational Publishers: Scranton, PA, 1972.

14. D. E. Rivera, M. Morari, and S. Skogestad, "Internal model control: PID controller design," Industrial \& Engineering Chemistry ProcessDesign and Development, vol. 25, pp. 252265, 1986. 